\title{
Winfried „Mustapha“ Müller und der algerische Unabhängigkeitskrieg
}

\author{
Moritz Oberhollenzer \\ Kerngebiet: Zeitgeschichte \\ eingereicht bei: Ass.-Prof. Mag. Dr. Eric Burton \\ eingereicht im: SoSe 2019 \\ Rubrik: Proseminar-Arbeit
}

\begin{abstract}
Winfried „Mustapha“ Müller and the Algerian War of Independence

This paper is about the involvement of Winfried "Mustapha" Müller in the Algerian War of Independence from 1954 to 1962. It will focus on how his work for the FLN (Front de Libération Nationale) helped in the struggle for Algerian independence from the French motherland. In this context it incorporates a transnational perspective on how the war could be won not only by the fighters of the FLN, but also by people fuelling the international discussion talking about the war.
\end{abstract}

\section{Einleitung}

Im November des Jahres 1954 brach der algerische Unabhängigkeitskrieg aus. ${ }^{1}$ Es sollte acht Jahre dauern, bis Algerien schließlich die politische Unabhängigkeit vom französischen Mutterland errang. ${ }^{2}$ Je nach Quelle kamen in diesem Krieg zwischen 150.000

1 Daniel Panagiotopoulos, Das Leben in der Fremdenlegion nach 1945. Ein Oral-History-Projekt mit ExLegionären aus Nordtirol, Südtirol und Vorarlberg, Dipl. Innsbruck 2012, S. 43; Hartmut Elsenhans, Frankreichs Algerienkrieg 1954-1962. Entkolonialisierungsversuch einer kapitalistischen Metropole. Zum Zusammenbruch der Kolonialreiche, München 1974, S. 1; Andreas Feichter, Österrreich und der algerische Unabhängigkeitskrieg. Aspekte der Diskussion über "Nationale Befreiung“ im Österreich der fünfziger Jahre, Diss. Innsbruck 1988, S. 67; Mathias Günther Ritzi, Richard Christmann. Nachrichtendienstliche Auseinandersetzung zwischen Deutschland und Frankreich in den Jahren von 1936 bis 1961, Diss. Innsbruck 2010, S. 239.

2 Ritzi, Richard Christmann, S. 235; Feichter, Österreich und der algerische Unabhängigkeitskrieg, S. 67. 
und 1 Million Menschen ums Leben, den größeren Teil der Verluste erlitt die algerische Bevölkerung. ${ }^{3}$

Einer der algerischen Befreiungskämpfer war ab 1956 Winfried „Mustapha“ Müller. ${ }^{4}$ Bereits seit 1955 war er der FLN (Front de Libération Nationale) in Paris zur Hand gegangen. ${ }^{5}$ Durch den von ihm erdachten und geleiteten „Rückführungsdienst“ verhalf Müller zwischen den Jahren 1956 und 1962 etwa 4.000 in Algerien stationierten Fremdenlegionären zur Desertation und oft auch zurück in ihre Herkunftsländer. ${ }^{6}$

Über den Algerienkrieg selbst wurde bereits viel und ausführlich geschrieben. Als Beispiel soll hier nur das zweiteilige Werk von Hartmut Elsenhans angeführt werden, das sich genauestens mit der gesellschaftlichen, ökonomischen und politischen Situation in Algerien beschäftigt, mit der Bedeutung Algeriens für das französische Vaterland, mit den einzelnen Kampfhandlungen während des Krieges, mit den französischen Mitteln zur Wiederherstellung des Friedens, mit den Verhandlungen, die am Ende zur Unabhängigkeit Algeriens führten, und mit den Auswirkungen dieses Krieges auf das innere Frankreich. ${ }^{7}$ Vergleichsweise wenig wurde über die Rolle Winfried „Mustapha“ Müllers in diesem Krieg geschrieben, so wird er auch in Hartmut Elsenhans' Werk mit keinem Wort erwähnt. Dennoch hat sich die Zahl der über Winfried Müller verfassten Zeilen mit den Jahren auf ein doch ansehnliches Maß erhöht, wenngleich hier die Betonung auf dem Wort "Zeilen" liegt; denn häufig wird er bloß als Nebenakteur genannt - wie in Andreas Feichters und Mathias Ritzis Dissertationen, ${ }^{8}$ im Buch „Hoch die internationale Solidarität” von Werner Balsen und Karl Rössel, ${ }^{9}$ in "West Germany, Cold War Europe and the Algerian War" von Mathilde von Bülow ${ }^{10}$ und in Claus Leggewies Aufsatz „Kofferträger"11. Dennoch gibt es auch Schriften, die Winfried Müller in den Mittelpunkt stellen, so zum einen den Aufsatz Klaus Polkehns "Die Mission des Si Mustapha - ein Deutscher kämpft für Algerien", 12 zum anderen die 2018 erschienene Biografie Fritz Kellers mit dem Titel „Ein Leben am Rande der Wahrscheinlichkeit", 13 in der sich Keller mit der ausführlichen Erkundung des undurchsichtigen Lebens Winfried Müllers befasst.

3 Elsenhans, Frankreichs Algerienkrieg, S. 534; Douglas Boyd, Die französische Fremdenlegion, Hamburg-BerlinBonn 2006, S. 73.

4 Fritz Keller, Ein Leben am Rande der Wahrscheinlichkeit. Si Mustapha alias Winfried Müller. Vom Wehrmachtsdeserteur zum Helden des algerischen Befreiungskampfes, Wien 2017, S. 45; Feichter, Österreich und der algerische Unabhängigkeitskrieg, S. 85.

5 Keller, Leben am Rande der Wahrscheinlichkeit, S. 41; Claus Leggewie, Kofferträger. Das Algerien-Projekt in den 50er und 60er Jahren und die Ursprünge des "Internationalismus" in der Bundesrepublik, in: Politische Vierteljahrsschrift 25 (1984), Nr. 2, S. 169-187, hier S. 179; Klaus Polkehn, Die Mission des Si Mustapha. Ein Deutscher kämpft für Algerien, in: COMPARATIV 16 (2006), Heft 2, S. 30-45, hier S. 33; Feichter, Österreich und der algerische Unabhängigkeitskrieg, S. 85.

6 Polkehn, Die Mission des Si Mustapha, S. 42; Leggewie, Kofferträger. Das Algerien-Projekt in den 50er und 60er Jahren, S. 179-180; Feichter, Österreich und der algerische Unabhängigkeitskrieg, S. 110.

7 Elsenhans, Frankreichs Algerienkrieg.

8 Ritzi, Richard Christmann; Feichter, Österreich und der algerische Unabhängigkeitskrieg.

9 Werner Balsen/Karl Rössel, Hoch die internationale Solidarität. Zur Geschichte der Dritte-Welt-Bewegungen in der Bundesrepublik, Köln 1986.

10 Mathilde von Bülow, West Germany, Cold War Europe and the Algerian War, Cambridge 2016.

11 Leggewie, Kofferträger. Das Algerien-Projekt in den 50er und 60er Jahren.

12 Polkehn, Die Mission des Si Mustapha.

13 Keller, Leben am Rande der Wahrscheinlichkeit. 
Diese Arbeit soll sich in die Liste der Schriften zu Winfried Müller einreihen, beschäftigt sie sich doch mit der Frage, inwiefern Müllers Arbeit in seinem Rückführungsdienst dem algerischen Unabhängigkeitsbestreben gedient hat. Die These dieser Arbeit ist, dass sie nicht nur militärischen Nutzen hatte, sondern die neu entstehende Nation auch auf politischer und mentaler Ebene unterstützte; des Weiteren, dass der Nutzen auf politischer Ebene weitaus bedeutender für das Erlangen der algerischen Unabhängigkeit war als auf der militärischen. Dabei wird eine transnationale Perspektive auf den algerischen Befreiungskrieg geworfen, der in ein komplexes System aus internationalen Beziehungen eingebunden war und durch dieses schließlich sein Ende fand. Es soll in dieser Arbeit im Kontext der transnationalen Perspektive gezeigt werden, wie Müller durch seine Tätigkeit als Leiter des Rückführungsdienstes einen Beitrag zum Herbeiführen dieses Kriegsendes leisten konnte.

\section{Winfried „Mustapha“ Müller und die FLN}

Winfried Müller wurde im Jahr 1926 als Sohn Curt Willi Ernst Müllers und Friederike Katherine Neelsons in Wiesbaden geboren und verbrachte seine Kindheit in Oberstdorf in der Nähe der deutsch-österreichischen Grenze. ${ }^{14}$ Andreas Feichter schreibt in seiner Dissertation, Müller sei bereits 1932 zu Verwandten in Götzens in Tirol geschickt worden. ${ }^{15}$ Keller und Polkehn behaupten demgegenüber, dass Winfried Müller gemeinsam mit seiner Mutter, die seinen Vater bereits kurz nach der Machtergreifung Hitlers verlassen habe, erst 1941 nach Götzens übersiedelt sei. ${ }^{16}$

1943 wird Müller von der Gestapo verhaftet, ${ }^{17}$ wahrscheinlich, weil er nazifeindliche Radiosender hörte. ${ }^{18}$ Allerdings variiert das Vergehen je nach Quelle, so ist bei Keller auch von einer anderen Version der Geschichte die Rede, in welcher Müller wegen "Österreichisch-patriotischen Parolen an öffentlichen Plätzen“ festgenommen worden sei. ${ }^{19}$ Feichter erzählt gar von Müllers „Aktivitäten im österreichischen Widerstand“20. So ist in den verschiedenen Versionen auch von unterschiedlichen Bestrafungen die Rede: In den beiden letzteren Darstellungen wurde Müller in ein Konzentrationslager eingeliefert. ${ }^{21}$ Aus diesem konnte er entweder nach sechs Monaten in Gefangenschaft in die Sowjetunion fliehen ${ }^{22}$ oder er wurde durch einen SS-Mann in ein Strafbataillon an die Ostfront geschickt. ${ }^{23}$ Aus jenem konnte er später in russische Reihen desertieren. ${ }^{24}$ In der erstgenannten (aufgrund jüngeren Forschungsstands wahrscheinlicheren) Version

\footnotetext{
14 Keller, Leben am Rande der Wahrscheinlichkeit, S. 9.

15 Feichter, Österreich und der algerische Unabhängigkeitskrieg, S. 83.

16 Keller, Leben am Rande der Wahrscheinlichkeit S. 9 und 11; Polkehn, Die Mission des Si Mustapha, S. 31.

17 Ebd., S. 17; Feichter, Österreich und der algerische Unabhängigkeitskrieg, S. 84; Polkehn, Die Mission des Si Mustapha, S. 31.

18 Keller, Leben am Rande der Wahrscheinlichkeit, S. 17; Polkehn, Die Mission des Si Mustapha, S. 31.

19 Keller, Leben am Rande der Wahrscheinlichkeit, S. 15.

20 Feichter, Österreich und der algerische Unabhängigkeitskrieg, S. 84.

21 Ebd.; Keller, Leben am Rande der Wahrscheinlichkeit, S. 15.

22 Feichter, Österreich und der algerische Unabhängigkeitskrieg, S. 84.

23 Keller, Leben am Rande der Wahrscheinlichkeit, S. 16.

24 Claus Leggewie, Kofferträger. Das Algerienprojekt der Linken im Adenauer-Deutschland, Berlin 1984, S. 89, zit. nach Polkehn, Die Mission des Si Mustapha, S. 31.
} 
meldete er sich freiwillig zum Militärdienst und wurde erst in ein Strafbataillon gesteckt - aus dem er später wiederum in russische Reihen desertieren konnte, als er "zunehmende, offene Widersetzlichkeit [...] gegenüber der Militär-Maschinerie“" zeigte. ${ }^{25}$

Nach der Desertation arbeitete Müller für das Nationalkomitee Freies Deutschland (NKFD) ${ }^{26}$ Es handelte sich um eine Gruppe deutscher Kriegsgefangener kommunistischer Gesinnung, die versuchte, durch Propagandaarbeit deutsche Soldat*innen zum Überlaufen auf die russische Seite zu bewegen. ${ }^{27}$ Hier kam er mit dem Konzept, dem später auch sein Rückführungsdienst während des algerischen Unabhängigkeitskrieges folgen sollte, wahrscheinlich erstmals in Kontakt.

Auf den Krieg folgten unruhige Jahre: Zunächst hielt er sich kurz in Österreich auf.28 Anschließend übersiedelte er 1947 nach Kleinmachnow in der Sowjetischen Besatzungszone, wurde im Jänner 1948 SED-Mitglied und studierte "Soziologie und Verhaltenslehre" auf der Parteihochschule „Karl Marx" in der Hakeburg bei Kleinmachnow. ${ }^{29}$ Womöglich kam ihm dieses Studium später in seiner Tätigkeit als Leiter des Rückführungsdienstes zu Hilfe. 1951 wurde er aus der Partei ausgeschlossen. Es besteht die Möglichkeit, dass er bereits ab 1948 für amerikanische Geheimdienste arbeitete; so wurde inm bei seinem Ausschluss aus der Partei vorgeworfen, ein "Agent und Provokateur" zu sein. Es wurde ihm vorgeworfen, eine Mitgliederliste der FDJ Wiesbaden sei durch ihn an den amerikanischen Geheimdienst gelangt. ${ }^{30}$ In weiterer Folge schloss Müller Kontakte zur Unabhängigen Arbeiterpartei Deutschlands. ${ }^{31}$

Dann ging er nach Paris. Keller zufolge war er vom Geheimdienst der USA dorthin geschickt worden. ${ }^{32}$ Wiederum laut Keller hatte er bereits vor dem Beginn seiner Kontakte zur FLN in Paris die Idee für seinen späteren Rückführungsdienst - genauer: nachdem er von "US-amerikanischen Quellen“ in einer Villa in Nikolassee (Berlin) vom Algerienkrieg erfahren hatte. ${ }^{33}$ So habe Müller sich schon im Jahr 1955 in Paris daran gemacht, Fremdenlegionäre zur Desertation zu bewegen. ${ }^{34}$ Damit einhergehend transportierte er wahrscheinlich Waffen für die FLN. ${ }^{35}$ Bald kam es zur Denunziation gegenüber französischen Geheimdiensten. ${ }^{36}$ Müller musste auf Anordnung der Polizei bis Ende 1956 Frankreich verlassen. ${ }^{37}$ So fuhr er, wohl nach Absprache mit der FLN, nach Marokko, wo er möglicherweise in ein Ausbildungslager der ALN (Armée de libération na-

25 Keller, Leben am Rande der Wahrscheinlichkeit, S. 17-19.

26 Ebd., S.19-20; Polkehn, Die Mission des Si Mustapha, S. 31; Feichter, Österreich und der algerische Unabhängigkeitskrieg, S. 84.

27 Keller, Leben am Rande der Wahrscheinlichkeit, S. 21.

28 Ebd., S. 22.

29 Feichter, Österreich und der algerische Unabhängigkeitskrieg, S, 84; Keller, Leben am Rande der Wahrscheinlichkeit, S. 24.

30 Ebd., S. 26-28; Polkehn, Die Mission des Si Mustapha, S. 32.

31 Polkehn, Die Mission des Si Mustapha, S. 32-33.

32 Keller, Leben am Rande der Wahrscheinlichkeit, S. 39.

33 Ebd., S. 37-38.

34 Ebd., S. 41.

35 Ebd.; Polkehn, Die Mission des Si Mustapha, S. 33.

36 Feichter, Österreich und der algerische Unabhängigkeitskrieg, S. 85

37 Keller, Leben am Rande der Wahrscheinlichkeit, S. 41. 
tionale; militärischer Arm der FLN) kam. ${ }^{38}$ Auf jeden Fall pflegte er aber Kontakte zu dieser. ${ }^{39}$ Wahrscheinlich ließ sich Müller aufgrund seiner politischen Überzeugung auf die Zusammenarbeit mit der FLN ein und im Oktober 1956 schrieb er an seine Frau, er habe "erstmals seit dem Zusammenbruch des falschen kommunistischen Himmels die direction gefunden"40. Wie sehr er sich für den Kampf der FLN begeisterte, zeigt auch, dass er, in Algerien angekommen, zum Islam konvertierte und dabei den Namen "Mustapha” annahm. ${ }^{41}$ Polkehn schreibt hierzu: "Er bekannte sich zu dem Ziel, ,daß ich Algerier werde und Algerier bleibe."'42

Die FLN wusste zunächst nicht recht, wie Müller ihrem Unabhängigkeitskampf dienen sollte. ${ }^{43}$ Erst im Herbst 1956 fand sich Arbeit für ihn, als er als Dolmetscher für eine Gruppe gefangener deutscher Fremdenlegionäre fungieren sollte. ${ }^{44}$ Bei dieser Gelegenheit sprach Müller von seiner Idee, einen Rückführungsdienst für desertierte Fremdenlegionäre zu gründen. Der Nationalrat der algerischen Unabhängigkeitsbewegung (CNRA) bewilligte diesen im Oktober desselben Jahres. ${ }^{45}$ Seine Aufgabe bestand in erster Linie darin, Fremdenlegionäre von der Desertation zu überzeugen und sie anschließend durch Kontaktaufnahme mit den Botschaften ihrer jeweiligen Heimatländer sicher in diese zurückzubringen. ${ }^{46}$ Sein Ziel war es erstens, die „Sensibilisierung der öffentlichen Meinung" zu bewirken, zweitens, die „Umwandlung einer Eliteeinheit in einen Unsicherheitsfaktor für den Feind“ zu erreichen, und drittens, „Mittel zur Anerkennung der Revolution auf internationaler Ebene" zu sein. ${ }^{47}$ Wie diese Ziele erreicht wurden, wird in den folgenden Kapiteln gezeigt werden.

Die FLN, in deren Diensten Müller nun stand, war zu Beginn des Krieges im November 1954 aus dem CRUA (Comité Revolutionnaire d'Unité et d'Action) hervorgegangen. ${ }^{48}$ Dieses wurde wiederum als militärischer Verband zur Bekämpfung der französischen Kolonialherrschaft Anfang desselben Jahres von Mitgliedern der OS (Organisation Secréte) gegründet. ${ }^{49}$ Die OS war nach dem Zweiten Weltkrieg gegründet worden, um den „bewaffneten Aufstand“ vorzubereiten. ${ }^{50}$ Die FLN bzw. die ALN hatten am 1. November 1954 mit einer sich über ganz Algerien erstreckenden Attentatswelle auf zivile wie militärische Ziele den algerischen Unabhängigkeitskrieg gestartet. ${ }^{51}$ Seither gingen alle

38 Polkehn, Die Mission des Si Mustapha, S. 33; Feichter, Österreich und der algerische Unabhängigkeitskrieg, S. 85.

39 Keller, Leben am Rande der Wahrscheinlichkeit, S. 44.

40 Ebd., S. 46; Polkehn, Die Mission des Si Mustapha, S. 34.

41 Keller, Leben am Rande der Wahrscheinlichkeit, S. 46; Polkehn, Die Mission des Si Mustapha, S. 34.

42 Polkehn, Die Mission des Si Mustapha, S. 34.

43 Ebd., S. 33.

44 Keller, Leben am Rande der Wahrscheinlichkeit, S. 45.

45 Ebd.; Polkehn, Die Mission des Si Mustapha, S. 34-35.

46 Keller, Leben am Rande der Wahrscheinlichkeit, S. 48.

47 Feichter, Österreich und der algerische Unabhängigkeitskrieg, S. 90-91; Keller, Leben am Rande der Wahrscheinlichkeit, S. 45-46.

48 Feichter, Österreich und der algerische Unabhängigkeitskrieg, S. 63; Ritzi, Richard Christmann, S. 239.

49 Ritzi, Richard Christmann, S. 238.

50 Feichter, Österreich und der algerische Unabhängigkeitskrieg, S. 62.

51 Elsenhans, Frankreichs Algerienkrieg, S. 150; Feichter, Österreich und der algerische Unabhängigkeitskrieg, S. 63; Panagiotopoulos, Leben in der Fremdenlegion nach 1945, S. 43. 
anderen nationalistischen Bewegungen - bis auf Teile des MNA, die 1957 folgen sollten - in ihr auf oder wurden liquidiert.52

Der von der ALN geführte Guerillakrieg war zu Beginn des Krieges noch auf das AurésGebirge und die Kabylei konzentriert gewesen, hatte sich im August 1955 allerdings auch auf die nördliche Städtelandschaft ausgeweitet..$^{53} \mathrm{Im}$ September 1956 kontrollierte die ALN bereits vier Fünftel des algerischen Landes. ${ }^{54}$ Im Mai 1955 waren erstmals französische Militärverbände von der ALN direkt angegriffen worden. ${ }^{55}$ Während die ALN zu Beginn des Krieges nur etwa 3.000 kämpfende Mitglieder zählte, waren es Ende des Jahres 1956 bereits um die 20.000.56 Gleichzeitig wurde sie von großen Teilen der nicht-französischen Bevölkerung unterstützt. ${ }^{57}$ Auch in Sachen Bewaffnung hatte die ALN bis 1957 eine solide Basis aufgebaut: Elsenhans spricht von „700 - 800 moderne[n] Waffen", die monatlich für den algerischen Unabhängigkeitskampf geliefert worden

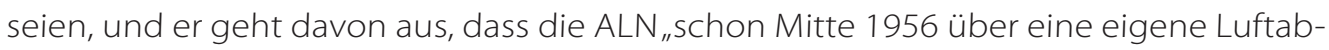
wehr verfügte" 58 .

So fand sich Müller zur Zeit der Gründung des Rückführungsdienstes in einer politisch wie militärisch stabilen Revolution wieder.

\section{Der militärische Nutzen}

Zu Beginn des algerischen Unabhängigkeitskrieges war die französische Stellung in Algerien durch den gerade erst beendeten Indochinakrieg stark geschwächt. Auf die Berufsarmee war kein Verlass, war sie doch durch die erlittenen Verluste stark geschrumpft und zu großen Teilen nicht in Algerien stationiert. ${ }^{59}$ So zählten die französischen Truppen in Algerien am 1. November 1954 nur 62.000 Personen, wobei ein großer Teil der französischen Elitetruppen sich eben nicht in Algerien aufhielt. ${ }^{60}$ Von der Fremdenlegion war allein das $1^{\mathrm{e}} \mathrm{REI}$ (régiment étranger d'infanterie) einsatzfähig. ${ }^{61}$ Bis zum Herbst 1956 sollte sich die militärische Situation aber drastisch verändern. Durch einige Änderungen im Bereich der Wehrpflicht (das Dienstalter wurde verringert, die Dienstdauer verlängert) und die Verlegung von Regimentern erreichte das französische Heer in Algerien zu dieser Zeit eine Truppenstärke von 360.000, die sich bis zum Ende des Krieges nicht sonderlich erhöhen sollte. ${ }^{62}$ Die Fremdenlegion hatte im Jahr 1956 ihren Höchststand von 27.500 Mann erreicht. ${ }^{63}$ Gemeinsam mit den Fallschirmjägereinheiten der regulären französischen Armee hatte die Fremdenlegion eine besondere Bedeutung, da sie bis 1957, als dann auch neu einberufene Wehrpflichtige

52 Elsenhans, Frankreichs Algerienkrieg, S. 152-153.

53 Ritzi, Richard Christmann, S. 240.

54 Elsenhans, Frankreichs Algerienkrieg, S. 379.

55 Ebd.

56 Ebd, S. 380.

57 Ebd., S. 380-381; Feichter, Österreich und der algerische Unabhängigkeitskrieg, S. 70.

58 Elsenhans, Frankreichs Algerienkrieg, S. 381.

59 Ebd., S. 391-392.

60 Ebd.

61 Boyd, Französische Fremdenlegion, S. 53.

62 Elsenhans, Frankreichs Algerienkrieg, S. 393-395; Ritzi, Richard Christmann, S. 240.

63 Panagiotopoulos, Leben in der Fremdenlegion nach 1945, S. 47. 
eine spezielle Ausbildung erfuhren, als einziger Truppenverband für den Guerillakrieg in Algerien ausgebildet war. ${ }^{64}$ Zudem hatte sie in der französischen Armee den Status einer Eliteeinheit, die dort eingesetzt wurde, „wo sich die reguläre Armee weigerte zu intervenieren [...] oder das Risiko (für diese) zu groß war" ${ }^{\prime \prime 5}$.

Müllers Rückführungsdienst fand seine Heimat in Tetuan im früheren Spanisch-Marokko. ${ }^{66}$ Dort wurde der algerischen Befreiungsbewegung von der marokkanischen Befreiungsarmee eine Villa als Basis für Müllers Arbeit zur Verfügung gestellt.67 In dieser Villa fanden die desertierten Fremdenlegionäre Unterschlupf, bis sie (zumeist) in ihre jeweiligen Heimatländer zurückgebracht werden konnten. ${ }^{68} \mathrm{Um}$ die Fremdenlegionäre von der Desertation zu überzeugen, ließ Müller Poster und Flugblätter anfertigen, deren Aufschrift meist etwas wie „Entweder im Heimatland leben oder in der Legion sterben“ aussagte und auf denen Bilder von bereits geflohenen Fremdenlegionären in ihren Heimatländern zu sehen waren. ${ }^{69}$ Es kam aber auch zu persönlichen Aufforderungen an Fremdenlegionäre durch Agenten der FLN, sich von der Legion zu verabschieden. ${ }^{70}$ Außerdem wurden persönliche Briefe vom Rückführungsdienst selbst oder von helfenden Personen an verschiedene Fremdenlegionäre gesandt. So kam es beispielsweise dazu, dass Fremdenlegionäre nach der brieflichen Verabredung mit einer "jungen Frau“, die Interesse an einem Treffen mit einem Legionär zeigte, ein Mitglied des Rückführungsdienstes am verabredeten Treffpunkt vorfanden. ${ }^{71}$ Ab dem Frühjahr 1957 wurde dem Rückführungsdienst von Seiten der DDR sogar ein eigener Radiosender zur Verfügung gestellt, über den Fremdenlegionäre von der Desertation überzeugt werden sollten. ${ }^{72}$

Ein Punkt, der die Legionäre zur Desertation brachte, war wohl ihre geringe Überlebenschance in Algerien. Einige der Legionäre waren außerdem durch die Niederlagen und Entbehrungen im Indochinakrieg demoralisiert. ${ }^{73}$ Auch waren nicht alle freiwillig der Legion beigetreten; einige waren unter Vorspiegelung falscher Tatsachen rekrutiert worden oder waren sich bei ihrem Beitritt nicht über alle Konsequenzen ihrer Entscheidung im Klaren gewesen. ${ }^{74}$ Manche „Deserteure“ wurden allerdings auch gewaltsam überwältigt und anschließend über die Grenze nach Tetuan gebracht, dort angekommen war ihnen der Rückweg versperrt. Die Legion war ihnen gegenüber nach ihrem Verschwinden naturgemäß misstrauisch. ${ }^{75}$

64 Elsenhans, Frankreichs Algerienkrieg, S. 401-403.

65 Mustapha Müller, in: Ministere de la Culture et du Tourisme (Hrsg.), Konferenzprotokoll „Le Service de Rapatriement des legionnaires Etrangers", Algiers 1986, zit. nach Feichter, Österreich und der algerische Unabhängigkeitskrieg, S. 83 .

66 Keller, Leben am Rande der Wahrscheinlichkeit, S. 48-49; Polkehn, Die Mission des Si Mustapha, S. 34

67 Keller, Leben am Rande der Wahrscheinlichkeit, S. 48-49.

68 Ebd., S. 55

69 Ebd., S. 50-54; Polkehn, Die Mission des Si Mustapha, S. 37; Feichter, Österreich und der algerische Unabhängigkeitskrieg, S. 94-96.

70 Keller, Leben am Rande der Wahrscheinlichkeit, S. 51-52.

71 Feichter, Österreich und der algerische Unabhängigkeitskrieg, S. 92-93.

72 Ebd., S. 71; Polkehn, Die Mission des Si Mustapha, S. 40.

73 Keller, Leben am Rande der Wahrscheinlichkeit, S. 52-53.

74 Ebd., S. 61-62; Polkehn, Die Mission des Si Mustapha, S. 39

75 Keller, Leben am Rande der Wahrscheinlichkeit, S. 52. 
So gelang es dem Rückführungsdienst innerhalb kürzester Zeit, die Hälfte des in Fort Tachtouf an der algerisch-marokkanischen Grenze stationierten $4^{\mathrm{e}} \mathrm{REI}$ zur Desertation zu bewegen. Die französische Armee war gezwungen, diesen wichtigen Stützpunkt aufzugeben, bevor noch weitere ihrer Soldat*innen dort verschwanden. ${ }^{76}$ Bis zum Ende des Krieges soll Müllers Rückführungsdienst nach seiner eigenen Aussage etwa 4.000 französische Fremdenlegionäre zur Fahnenflucht überredet haben, im Abschlussbericht des Rückführungsdienstes ist von 4.111 die Rede.77 Da beide Aussagen über die Zahl der desertierten Legionäre erst nach dem Ende des algerischen Unabhängigkeitskrieges erfolgt sind, sind sie vermutlich nicht absichtlich verfälscht, hatte der Rückführungsdienst doch zu diesem Zeitpunkt seine Aufgabe bereits erfüllt. Das mag bei Betrachtung der etwas mehr als 360.000 in Algerien stationierten Soldat*innen nicht als sonderlich viel erscheinen; allerdings wurde oben bereits angesprochen, dass die Fremdenlegion im Algerienkrieg von besonderer Bedeutung war. Und während 1956 noch die erwähnten 27.500 Fremdenlegionäre in Algerien stationiert waren, war ihre Zahl in den Jahren 1958 bis 1961 auf 21.000 Mann geschrumpft. ${ }^{78}$ Somit hatte der Rückführungsdienst zum einen auf operationeller Ebene Erfolg und erreichte hier sein Ziel, die Fremdenlegion in einen „Unsicherheitsfaktor für den Feind"79 zu verwandeln, wie der Fall von Fort Tachtouf zeigt. Zum anderen aber war es auch ein Erfolg auf einer höheren Ebene, indem er seinen Teil zur Verringerung der in Algerien stationierten Truppen beitrug.

Winfried Müllers Arbeit als Leiter des Rückführungsdienstes endete jedoch nicht bei dessen Verwaltung, er war auch in den Einkauf von Waffen für die ALN verwickelt.80 Dieser war für jene von äußerster Bedeutung. An möglichen Rekrut*innen mangelte es der ALN nämlich nicht, diese mussten aber mit Waffen versorgt werden, um sie als kampffähige Soldat*innen in die ALN aufnehmen zu können. Elsenhans schreibt hierzu: „Eine zahlenmäßige Vergrößerung der Befreiungsarmee [lag] weniger bei Schwierigkeiten der Rekrutierung als bei den Engpässen bei der Ausrüstung mit Waffen." ${ }^{11}$ Das genaue Ausmaß von Müllers Beteiligung an den Waffengeschäften ist noch nicht erforscht, laut Keller war er jedoch „einer der vier Hauptauftraggeber der FLN bei Waffengeschäften“82 Polkehn behauptet, dass Müller inm später voller Ärger davon erzählt habe, dass die DDR sich zwar dazu bereit erklärt habe, alte Weltkriegswaffen der Wehrmacht an die ALN zu liefern, dafür aber "exorbitante Preise in harten Devisen" verlangt habe. ${ }^{83}$ Zumindest der Ärger darüber lässt eine persönliche Beteiligung Müllers bei den Waffengeschäften als wahrscheinlich erscheinen. Diese Waffengeschäfte mit der DDR seien unter anderem 1960 bei Müllers Reise in die DDR abgewickelt worden,

76 Keller, Leben am Rande der Wahrscheinlichkeit, S. 52-53; Feichter, Österreich und der algerische Unabhängigkeitskrieg, S. 110-111.

77 Feichter, Österreich und der algerische Unabhängigkeitskrieg, S. 110; Polkehn, Die Mission des Si Mustapha, S. 42; Leggewie, Kofferträger. Das Algerien-Projekt in den 50er und 60er Jahren, S. 179-180.

78 Panagiotopoulos, Leben in der Fremdenlegion nach 1945, S. 47.

79 Keller, Leben am Rande der Wahrscheinlichkeit, S. 46; Feichter, Österreich und der algerische Unabhängigkeitskrieg, S. 91.

80 Keller, Leben am Rande der Wahrscheinlichkeit, S. 80; Polkehn, Die Mission des Si Mustapha, S. 41.

81 Elsenhans, Frankreichs Algerienkrieg, S. 380.

82 Keller, Leben am Rande der Wahrscheinlichkeit, S. 80.

83 Polkehn, Die Mission des Si Mustapha, S. 41-42. 
als er unter anderem die Zusammenarbeit zwischen Rückführungsdienst und DDR verlängern wollte. ${ }^{84}$ So ist Müllers Hilfe für die FLN als Akteur im Waffenhandel nicht im Detail rekonstruierbar, jedoch zumindest als ein Teilbereich seiner Tätigkeit feststellbar.

Die bisherigen Ausführungen haben gezeigt, inwiefern Müllers Arbeit im Rückführungsdienst den Kampfhandlungen der FLN in Algerien dienstbar war. Bei Betrachtung des Kriegsverlaufs ist allerdings erkennbar, dass diese den Krieg allein auf militärischer Ebene nicht gewinnen konnte. Die ALN erhöhte bis in den Herbst 1956 ihre Zahl auf etwa 20.000 Kämpfer*innen. ${ }^{85}$ Die Stärke der französischen Armee wuchs auf die erwähnten 360.000 und sollte im Sommer 1957 ihren Höchststand von 396.000 Personen erreichen. ${ }^{86}$ Diese große Anzahl an Soldat*innen machte die Anlegung eines dichten Netzwerkes aus Militärstützpunkten möglich, die ihr jeweiliges Umland verteidigen sollten, wodurch die Verluste der ALN in die Höhe schossen. ${ }^{87}$ Die ALN konnte ihre Truppengröße jedoch halten und bis zum Jahr 1958 sogar auf 25.000 Kämpfer*innen erhöhen, ${ }^{88}$ Zahl und Dimension der Anschläge der FLN sanken aber während des Jahres 1957, zu dessen Beginn sie ihren Höhepunkt erreicht hatten, stark ab. ${ }^{89}$

Das Jahr 1958 kann als Schlüsseljahr des Krieges angesehen werden. In den Jahren 1956 und 1957 waren an den Grenzen zu Marokko und zu Tunesien Grenzbefestigungen angelegt worden.90 Diese zeigten ab Jahreswechsel 1957/58 ihre Wirkung:91 Während nach französischen Schätzungen im Januar 1958 erst fünfzig Prozent der an die FLN gelieferten Waffen abgefangen werden konnten, waren es im März bereits neunzig Prozent. ${ }^{92}$ Auch wurde Ende des Jahres 1958 General Salan ${ }^{93}$ durch General Challe ${ }^{94}$ ausgetauscht, wodurch ein Wechsel in der Taktik der französischen Armee stattfand..$^{95}$ Dieser entpuppte sich als militärisch äußerst erfolgreich. ${ }^{96}$ So sank die Zahl der Kämpfer*innen der ALN bis zum Ende des Krieges auf 2.000 bis 3.000 Mann. ${ }^{97} \mathrm{El}-$ senhans spricht daher von der "Niederlage der Guerilla auf militärischem Gebiet in Algerien“98. Feichter hingegen meint, dass der Krieg „Von der französischen Armee bereits zu diesem Zeitpunkt, dem Jahr 1956, nicht mehr gewonnen werden konnte" ${ }^{\prime 99}$. Der Sieg der Befreiungskämpfer*innen konnte aber nicht auf militärischer Ebene errungen

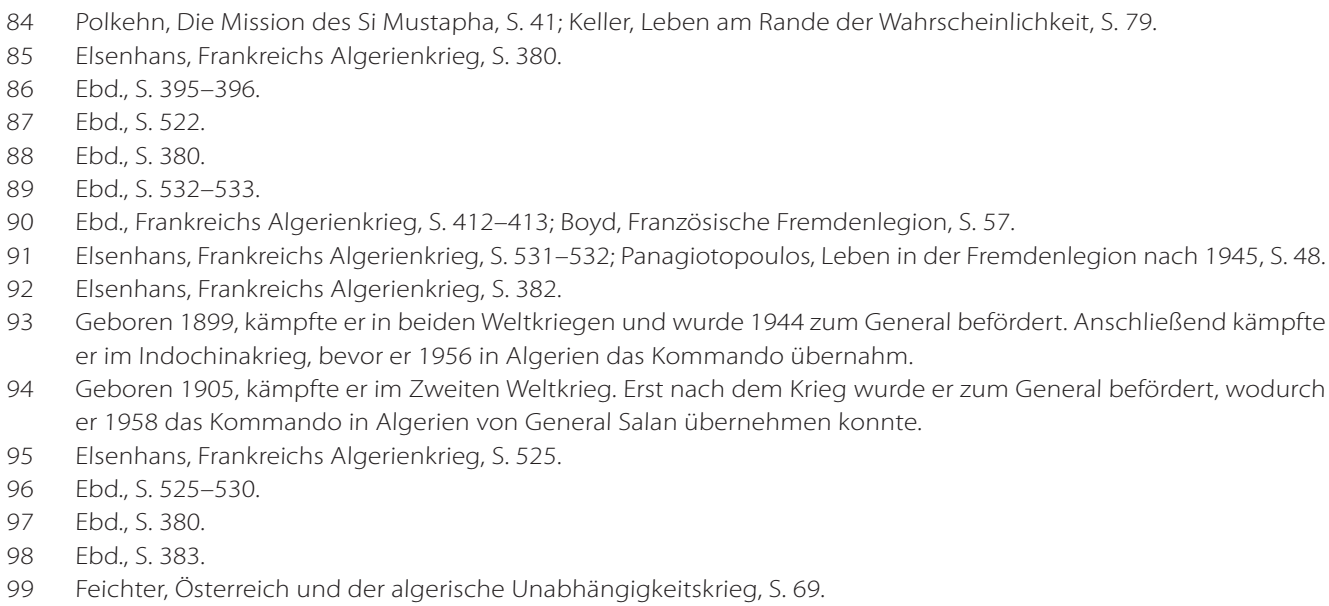


werden, sondern (nur) auf politischer. Um den Kampf auf dieser Ebene zu unterstützen, nutzte Müller seinen Rückführungsdienst auch zu Propagandazwecken.

\section{Der politische Nutzen}

Die rückgeführten Fremdenlegionäre sollten hierzu in ihren Heimatländern öffentlich machen, welche „Repressalien und Folterungen" sie selbst im Auftrag der französischen Armee begangen hatten, oder welche von ihnen beobachtet worden waren. ${ }^{100}$ Leggewie schreibt hierzu:

"Müller und seine Kontaktleute informierten die Presse, wenn eine Gruppe repatriierter Legionäre von Madrid nach Rhein-Main zurückkehrte, wo sie mit noch kommißkurzen Haaren, Schlips und kariertem Jackett wie Staatsgäste im Blitzlichthagel die Gangway hinunterstiegen."101

Desertierte ungarische Fremdenlegionäre, die nach der Zerschlagung des ungarischen Volksaufstandes 1956 nach Frankreich geflohen waren, konnten darüber berichten, wie sie einen Vertrag der Fremdenlegion unterschrieben hatten, um als Arbeiter in Algerien Anstellung zu finden. Ihnen war verschwiegen worden, dass sie eigentlich angeworben wurden, um auf dem Kriegsschauplatz in Algerien zu kämpfen. ${ }^{102}$ Müller schaffte es, die Geschichte der ungarischen Flüchtlinge sowohl in der internationalen Presse als auch in den diplomatischen Kreisen der UNO öffentlich zu machen, was Frankreich wenig Sympathie einbrachte, im Gegenteil. ${ }^{103}$

In der Presse war Müller vor allem im deutschsprachigen Raum präsent, wohl auch deshalb, weil die meisten der in Algerien kämpfenden Fremdenlegionäre deutschsprachig waren. ${ }^{104}$ Er selbst lieferte ab 1957 das Material an die Journalisten Klaus Polkehn und Hans Otten. ${ }^{105}$ Jene informierten daraufhin in der "Wochenpost" in der DDR über den Algerienkrieg. ${ }^{106}$ Bereits im Februar 1957 berichtete die „Bild“-Zeitung von einer "geheime[n] Macht, die innerhalb von acht Wochen tausende Fremdenlegionäre aus ihren nord-afrikanischen Kasernen befreit hat". ${ }^{107} 1959$ sprach Müller selbst in einem Interview mit dem „Spiegel“ (Titel: „Wer desertiert, muß ,Alemani' rufen“) von den Aktionen des Rückführungsdienstes. ${ }^{108}$ Im Herbst desselben Jahres reiste Müller sogar auf einer Werbekampagne durch die BRD, über welche „well over fifty German newspapers" schrieben. ${ }^{109}$ Feichter und von Bülow schreiben zu Müllers Präsenz in der Presse, er habe Journalisten bzw. Journalistinnen nach Marokko eingeladen, um die Aussagen

100 Leggewie, Kofferträger. Das Algerienprojekt der Linken, S. 95, zit. nach Feichter, Österreich und der algerische Unabhängigkeitskrieg, S. 101. Die französische Armee hatte im Rahmen des Unabhängigkeitskrieges auch Folter zur Informationsbeschaffung angewandt: Panagiotopoulos, Leben in der Fremdenlegion nach 1945, S. 45-46. Ebd.

102 Keller, Leben am Rande der Wahrscheinlichkeit, S. 61-64; Feichter, Österreich und der algerische Unabhängigkeitskrieg, S. 103-104.

103 Keller, Leben am Rande der Wahrscheinlichkeit, S. 63.

104 Feichter, Österreich und der algerische Unabhängigkeitskrieg, S. 89.

105 Polkehn, Die Mission des Si Mustapha, S. 30; Keller, Leben am Rande der Wahrscheinlichkeit, S. 71-72.

106 Feichter, Österreich und der algerische Unabhängigkeitskrieg, S. 101; von Bülow, West Germany, S. 118.

107 Bild, 10.2.1957, zit. nach Keller, Leben am Rande der Wahrscheinlichkeit, S. 52-53.

108 Feichter, Österreich und der algerische Unahbängigkeitskrieg, S. 95; von Bülow, West Germany, S. 237.

109 Ebd. 
der geflüchteten Legionäre schnell zu verbreiten und damit das Ansehen Frankreichs international zu schwächen".110 Von Bülow berichtet des Weiteren von Kontakten Müllers nicht nur zur bereits genannten „Wochenpost”, sondern eben auch zur "Süddeutschen Zeitung", zur "Welt" und zum "Spiegel“.111

Allerdings wurden Informationen über den Algerienkrieg nicht allein durch die Presse verbreitet. Müller hatte bereits früh gemeinsam mit den Journalisten Hans-Peter Rullmann und Hans Karl Lindemann den Nordafrikanischen Club gegründet, welcher verschiedene über den Algerienkrieg informierende Broschüren druckte. ${ }^{112}$ Auf einer Pressekonferenz desselben lernte Müller den deutschen Aktivisten Klaus Vack kennen. ${ }^{113}$ Dieser hatte sich bislang bereits durch Flugblattaktionen für die algerische Unabhängigkeit eingesetzt. ${ }^{114}$ In weiterer Folge unterstützte er Müllers Rückführungsdienst und dessen Propagandaarbeit direkt. ${ }^{15}$ So führte Müllers Rückführungsdienst durch dessen Öffentlichkeitsarbeit zu einer "Sensibilisierung der ausländischen öffentlichen Meinung ${ }^{\prime 116}$. Dieser hatte damit ein weiteres seiner Ziele erreicht. ${ }^{117}$

Schließlich muss noch einmal angemerkt werden, dass die Rückführung der Fremdenlegionäre in ihre jeweiligen Heimatländer nur nach Kontaktaufnahme mit den Botschaften derselben erfolgen konnte. ${ }^{118}$ Ihre Staatsbürgerschaft hatten sie durch den Dienst in einem ausländischen Heer verloren. ${ }^{119}$ Hierdurch konnte die FLN zum einen auf internationaler Ebene tätig sein, zum anderen konnte sie zeigen, dass sie „im Stande war, auf alle politischen, diplomatischen und humanitären Aspekte der Revolution hinzuweisen und die daraus entstehenden Probleme zu lösen".120 So konnte der Rückführungsdienst "Mittel zur Anerkennung der Revolution auf internationaler Ebene" sein. ${ }^{121}$ Müller konnte somit auch sein drittes Ziel erreichen.

Am Ende musste die französische Regierung auf Druck der Weltöffentlichkeit eine Lösung für ihren Kolonialkrieg in Algerien finden. ${ }^{22}$ Panagiotopoulos schreibt: „Das Ziel [der FLN] wurde erreicht ohne einen militärischen Sieg davonzutragen. [...] Am 3. Juli 1962 erkannte Frankreich Algeriens Unabhängigkeit an."123 Damit hörte auch Müllers Rückführungsdienst auf zu existieren. ${ }^{124}$

110 von Bülow, West Germany, S. 118; Feichter, Österreich und der algerische Unabhängigkeitskrieg, S. 90.

111 von Bülow, West Germany, S. 118.

112 Ebd., S. 117-118.

113 Keller, Leben am Rande der Wahrscheinlichkeit, S. 76.

114 Klaus Vack, Si Mustafa, die Rote Hand und 4000 deutsche Deserteure, in: Werner Balsen/Karl Rössel (Hrsg.), Hoch die internationale Solidarität, Köln 1986, S. 75-78, hier S. 76.

115 Ebd., S. 76-77; von Bülow, West Germany, S. 237-238.

116 Keller, Leben am Rande der Wahrscheinlichkeit, S. 45-46; Feichter, Österreich und der algerische Unabhängigkeitskrieg, S. 90

117 Feichter, Österreich und der algerische Unabhängigkeitskrieg, S. 111

118 Keller, Leben am Rande der Wahrscheinlichkeit, S. 55.

119 Feichter, Österreich und der algerische Unabhängigkeitskrieg, S. 98-99.

120 Ebd., S. 91.

121 Keller, Leben am Rande der Wahrscheinlichkeit, S. 46; Feichter, Österreich und der algerische Unabhängigkeitskrieg, S. 91.

122 Panagiotopoulos, Leben in der Fremdenlegion nach 1945, S. 48.

123 Ebd., S. 50

124 Keller, Leben am Rande der Wahrscheinlichkeit, S. 90. 


\section{Schluss}

So kann festgehalten werden, dass Müllers Arbeit im Rückführungsdienst der FLN auf militärischer Ebene hilfreich war, konnte er doch Teile der französischen Fremdenlegion durch seinen Rückführungsdienst von der Desertation überzeugen, wodurch erstens die Zahl der französischen Elitekräfte in Algerien dahinschwand und zweitens die bislang so verlässliche Fremdenlegion zu einem „Unsicherheitsfaktor für die französische Armee" wurde. Außerdem konnte Müller auch als Akteur im Waffenhandel den Erhalt und Ausbau der ALN unterstützen.

Allerdings kann in Folge argumentiert werden, dass das französische Heer, als es sich von der Niederlage in Indochina erholt hatte, doch zu schlagkräftig war, um von den algerischen Guerillakämpfern besiegt werden zu können. Somit musste die Unabhängigkeit Algeriens auf politischer Ebene errungen werden. Müllers Arbeit in seinem Rückführungsdienst konnte den Weg Algeriens hin zur Unabhängigkeit aber auch auf dieser Ebene unterstützen.

Erstens konnte er durch Pressearbeit - mit der Unterstützung von Aktivisten bzw. Aktivistinnen - die "Sensibilisierung der öffentlichen Meinung" vor allem im deutschsprachigen Raum erreichen. Hier ist anzumerken, dass für die Zeit des Algerienkriegs aber keineswegs von einem so großen Engagement gesprochen werden kann, wie es später für die Zeit des Vietnamkriegs zu verzeichnen ist. Allerdings spricht Leggewie von einer "Algerien-Solidarität" als der "ur- und frühgeschichtliche[n] Schicht des Internationalismus der westdeutschen Linken“125. Feichter schreibt in Bezug auf Österreich: „[Das] Engagement und die Solidarität breiterer Schichten in anderen Konflikten hatten doch ihren Ursprung in der Unterstützung für die algerische Revolution."126 Zweitens diente der Rückführungsdienst durch seine Arbeit auf internationaler diplomatischer Ebene zum Zweck der Rückführung der desertierten Fremdenlegionäre auch der „Anerkennung der Revolution auf internationaler Ebene".

Letztlich ist anzumerken, dass Winfried "Mustapha" Müller natürlich nur ein Rad in einem viel größeren Uhrwerk war; durch dessen Zusammenspiel allein konnte die algerische Nation schließlich unabhängig werden. Und es bleiben einige Fragen offen sowohl in Bezug auf Winfried Müllers Lebenslauf - viele davon werden wohl nie eindeutig beantwortbar sein - als auch in Bezug auf die Tätigkeit seines Rückführungsdienstes. Als Anregung für eine zukünftige Arbeit sollen hier nur zwei von ihnen genannt werden: Wie genau konnte der Rückführungsdienst die desertierten Fremdenlegionäre durch die Grenzbefestigungen an der algerisch-marokkanischen Grenze bringen? Und wie sah die Einbindung Winfried Müllers in den Waffeneinkauf der ALN im Detail aus?

Müller blieb nach der Auflösung seines Rückführungsdienstes zu Kriegsende in Algerien und versuchte, am Aufbau des nun unabhängigen Landes mitzuwirken. Er wurde allerdings von der Richtung, in die sich das Land bewegte, mehr und mehr enttäuscht und zog sich schließlich in die Bergregion der Kabylei zurück, wo er sich für die Grün- 
dung einiger Nationalparks einsetzte und seinen Lebensabend verbrachte. ${ }^{127}$ Während der Dreharbeiten an einem Dokumentarfilm über das Ahaggar-Gebirge erlitt er einen Herzinfarkt. Er starb am 9. Oktober 1993 in der südalgerischen Oase Tamanrasset und wurde in dem von inm gegründeten Tassilin-Nationalpark beerdigt. ${ }^{128}$

\section{Literatur}

Balsen, Werner/Rössel, Karl, Hoch die internationale Solidarität. Zur Geschichte der Dritte-Welt-Bewegung in der Bundesrepublik, Köln 1986.

Boyd, Douglas, Die französische Fremdenlegion, Hamburg-Berlin-Bonn 2009.

Bülow, Mathilde von, West Germany, Cold War Europe and the Algerian War, Cambridge 2016.

Elsenhans, Hartmut, Frankreichs Algerienkrieg 1954-1962, München 1974.

Feichter, Andreas, Österreich und der algerische Unabhängigkeitskrieg. Aspekte der Diskussion über „Nationale Befreiung“ im Österreich der fünfziger Jahre, Diss. Innsbruck 1988.

Keller, Fritz, Ein Leben am Rande der Wahrscheinlichkeit. Si Mustapha alias Winfried Müller. Vom Wehrmachtsdeserteur zum Helden des algerischen Befreiungskampfes, Wien 2017.

Leggewie, Klaus, Kofferträger. Das Algerien-Projekt in den 50er und 60er Jahren und die Ursprünge des „Internationalismus" in der Bundesrepublik, in: Politische Vierteljahrsschrift 25 (1984), Nr. 2, S. 169-187.

Ministere de la Culture et du Tourisme (Hrsg.), Konferenzprotokoll "Le Service de Rapatriement des Legionnaires Etrangers", Algiers 1986.

Panagiotopoulos, Daniel, Das Leben in der Fremdenlegion nach 1945. Ein Oral-HistoryProjekt mit Ex-Legionären aus Nordtirol, Südtiol und Vorarlberg, Dipl. Innsbruck 2012.

Polkehn, Klaus, Die Mission des Si Mustapha. Ein Deutscher kämpft für Algerien, in: COMPARATIV 16 (2006), Heft 2, S. 30-45.

Ritzi, Mathias Günther, Richard Christmann. Nachrichtendienstliche Auseinandersetzungen zwischen Deutschland und Frankreich in den Jahren von 1936 bis 1961, Diss. Innsbruck 2010.

Vack, Klaus, Si Mustafa, die Rote Hand und 4000 deutsche Deserteure, in: Werner Balsen/Karl Rössel (Hrsg.), Hoch die internationale Solidarität, Köln 1986, S. 75-78.

Moritz Oberhollenzer ist Student der Geschichtswissenschaften im 7. Semester an der Universität Innsbruck. moritz.oberhollenzer@student.uibk.ac.at 


\section{Zitation dieses Beitrages}

Moritz Oberhollenzer, Winfried „Mustapha“ Müller und der algerische Unabhängigkeitskrieg, in: historia.scribere 12 (2020), S. 107-120, [http://historia.scribere.at], eingesehen 15.6.2020 (=aktuelles Datum).

(C) Creative Commons Licences 3.0 Österreich unter Wahrung der Urheberrechte der Autorlnnen. 